\title{
Recent Advances in the Potential Use of Circular RNA for the Diagnosis and Treatment of Pancreatic Cancer
}

\author{
Xinzhu Sun' \\ Dongyan Liu ${ }^{2}$ \\ Nan $\mathrm{Ge}\left(\mathbb{D}^{\prime}{ }^{\prime}\right.$ \\ Jintao Guo' \\ Sheng Wang' \\ Xiang Liu' \\ Guoxin Wang' \\ Siyu Sun'
}

'Department of Gastroenterology, Shengjing Hospital of China Medical University, Shenyang, People's Republic of China; ${ }^{2}$ Department of Gastroenterology and Medical Research Center, Liaoning

Key Laboratory of Research and Application of Animal Models for Environmental and Metabolic Diseases, Shengjing Hospital of China Medical University, Shenyang, People's Republic of China
Correspondence: Siyu Sun

Department of Gastroenterology, Shengjing Hospital of China Medical University, 36 Sanhao Street, Shenyang, I 10004, People's Republic of China Email sunsy@sj-hospital.org

\begin{abstract}
There are few biomarkers available for the early diagnosis and prognostic evaluation of pancreatic cancer. In addition, the development of targeted therapy for pancreatic cancer is an unmet need due to the lack of molecular targets. With the continuous progress in circular RNA (circRNA)-related research, its role in the occurrence and development of pancreatic cancer has been discovered and gradually recognized. Therefore, circRNA may represent a novel marker for early diagnosis of this disease and a focus of targeted clinical therapy. CircRNA is a type of non-coding RNA with a closed circular structure formed by covalent bonds. Some circRNAs can act as "sponges" to adsorb microRNAs (miRNAs) and play the role of competitive endogenous RNA (ceRNA) to remove their inhibitory effects on the target genes of miRNA. Thus, they can indirectly restore the expression of target genes. The circRNA-miRNA-mRNA network plays a regulatory role in the proliferation, invasion, metastasis, and other biological behaviors of pancreatic cancer. Given the recent advances in circRNA, this review seeks to provide an overview of the biological function of circRNA and highlights the recent research progress regarding the molecular mechanism of circRNA for the clinical diagnosis and treatment of pancreatic cancer.
\end{abstract}

Keywords: circular RNA, pancreatic cancer, biological function, biomarker, targeted therapy

\section{Introduction}

Circular RNA (circRNA) is a covalently closed, endogenous biomolecule in eukaryotes that has tissue-specific and cell-specific expression patterns and is involved in various physiological and pathological processes. ${ }^{1}$ It was first discovered in advanced plants in the 1970 s as a pathogenic RNA molecule. ${ }^{2}$ Since then, it has been observed in the cytoplasm of eukaryotic cells and the mitochondria of yeast. ${ }^{3,4}$ With the rapid development of high-throughput sequencing, gene chip, and bioinformatics, many studies have demonstrated that circRNA is widely found in animals, plants, fungi, and protozoa. ${ }^{5}$ CircRNA is abundant in the cytoplasm of eukaryotic cells, ${ }^{6}$ whereas a small amount of intron or intron fragment-derived circRNA is present in the nucleus. ${ }^{7}$ CircRNA is more stable than linear RNA due to its closed-ring structure and lack of poly-A tails, which tend not to be degraded by exonucleases. $^{8}$ Homology studies among different species have shown that circRNA is highly conserved in the evolution of species. Its homology between mouse and pig is $15-20 \%{ }^{9}$ and between mouse and human is $20 \%$ or higher. $^{10}$ 
At first, most researchers believed that circRNA was a byproduct of mRNA splicing and possessed no significant biological function. ${ }^{6,11}$ However, advances in molecular biology and informatics have allowed researchers to determine that circRNA is widely present and expressed in eukaryotes ${ }^{10}$ and plays an important regulatory role in various vital activities and the pathogenesis and development of diseases. For example, aberrant circ-ITCH expression is related to aggressive clinicopathological features and unfavorable outcomes in various cancers. ${ }^{12}$ In addition, circRNA is highly conserved in sequence ${ }^{5}$ and stably expressed in various tissues and body fluids. Its expression levels vary in different tissues and growth stages. Thus, circRNA has a degree of tissue, temporal, and disease specificity that can be applied to disease prediction and evaluating the effect of diagnosis and treatment. ${ }^{13}$

Pancreatic cancer is a highly malignant tumor of the digestive system. Due to insidious initial symptoms, rapid development, and early distant metastasis, it is often difficult for patients to obtain an early diagnosis or timely treatment, leading to a very poor prognosis. The overall 5 -year survival rate is only about $8-9 \% .{ }^{14}$ Recently, the incidence and mortality of pancreatic cancer have increased year by year due to changes in dietary habits and lifestyle. Pancreatic cancer kills more than 200,000 people worldwide each year and is expected to be the second leading cause of cancer-related death by $2030 .{ }^{15}$ Due to the lack of specific targeted molecules, preoperative neoadjuvant chemotherapy and postoperative adjuvant therapy for pancreatic cancer fail to significantly improve the long-term survival rate of patients with pancreatic cancer. With the deepening research into the relationship between circRNA and pancreatic cancer, researchers have become aware that circRNA plays an important regulatory role in the development of this disease and may be a potential biomarker for early diagnosis and a therapeutic target.

In this review, the following aspects will be briefly introduced: classification and biogenesis of circRNA, the biological function of circRNA, and the research progress for circRNA in the molecular mechanism, clinical diagnosis, and treatment of pancreatic cancer.

\section{Overview of Circular RNA}

\section{Classification of Circular RNA}

CircRNA is mainly generated from exons that can encode proteins; however, some are derived from 3'-UTR, 5'-
UTR, introns, or intergenic spacer regions. ${ }^{16}$ CircRNA is produced by alternative splicing of special pre-messenger RNA, and the spliceosome plays an important role in its biogenesis. ${ }^{7}$ circRNA is mainly divided into three types according to its origin: 1) exon-derived circular RNA (exonic circRNA or ecircRNA), intron-derived circular RNA (intronic circRNA or ciRNA), ${ }^{17}$ and circular RNA composed of both exons and introns (retained-intron circRNA, exon-intron circRNA, or EIciRNA). ${ }^{18}$ Recently, tRNA intronic circRNA (tricRNA) was discovered, which is generated by pre-tRNA splicing. ${ }^{19,20}$

\section{Circular RNA Biogenesis EcircRNA Biogenesis}

EcircRNA is produced by back-splicing of pre-mRNA in which the $5^{\prime}$ splice donor of the downstream exon is bonded to the $3^{\prime}$ splice acceptor of the upstream exon, and a $3^{\prime}-5^{\prime}$ phosphodiester bond is created to form a circular structure. ${ }^{21}$ Jeck et $\mathrm{al}^{22}$ proposed two models for the mechanism of ecircRNA biogenesis. The first model, called "lariat-driven circularization", says that a circular formation is generated by the covalent combination of the $3^{\prime}$ and $5^{\prime}$ ends of the pre-mRNA exon. Subsequently, the introns are eliminated to generate the circRNA. The synthesis rate of this model is mainly affected by the upstream and downstream intron sequences. This process can competitively inhibit the linear splicing of pre-mRNA to regulate the transcription of related mRNA and the translation of proteins. ${ }^{23}$ The second model is called "intron-pairing-driven circularization". The two specific introns located on either side of the pre-mRNA exon form a hairpin structure through base complementation to enclose the specific exons and make adjacent exons spatially close to each other. The spliceosome then cuts the adjacent exons and the paired introns off to generate circRNA. Other studies have shown that some mature mRNA molecules previously spliced can be re-spliced and circularized into ecircRNA. ${ }^{24}$

\section{CiRNA Biogenesis}

CiRNA is synthesized by direct circularization of introns, and the specific mechanism is accomplished through a common motif. ${ }^{25}$ The $2^{\prime}$ end near the branching point of the motif contains a C-rich element of 11 nucleotides. A G-rich element composed of seven nucleotides is present near the $5^{\prime}$ splicing site. These two elements form a circular structure by complementary pairing, and the spliceosome cuts the exon and intron sequences off at 
the binding site. The introns are then connected to generate mature ciRNA.

\section{ElciRNA Biogenesis}

EIciRNAs are composed of exon and intron sequences from coding genes; that is, introns between the circularized exons remain. Therefore, EIciRNAs share the characteristics and functions of both exonic and intronic circRNA. The mechanisms of the biogenesis of EIciRNA and ecircRNA are possibly similar; ${ }^{18}$ however, the specific biogenesis mechanism for EIciRNA is not clear.

\section{TricRNA Biogenesis}

TricRNAs are a special type of intronic circRNA produced by tRNA precursors during splicing. ${ }^{26}$ Unlike the splicing of mRNA catalyzed by the spliceosome, splicing of tRNA occurs through tRNA splicing endonuclease (TSEN). TSEN is a heterotetramer composed of two binding groups and two catalytic groups ${ }^{27}$ that recognizes a bulge-helixbulge (BHB) motif located on tRNA precursors. It cuts the intron-containing tRNA precursors into three fragments $\left(5^{\prime}\right.$ exon, intron, and $3^{\prime}$ exon). Finally, tRNA ligase acts on the ends of the introns to produce tricRNA. ${ }^{19}$

\section{Biological Roles of Circular RNA CircRNA as a miRNA Sponge}

Some circRNAs can act as "sponges" to adsorb microRNA (miRNA) and remove the inhibitory effect of the miRNA on its target genes, ${ }^{28}$ indirectly leading to upregulated target gene expression. ${ }^{29,30}$ circRNA contains the reverse sequence of parental genes so that it competes with the latter for microRNA binding sites. Because of the lack of free $3^{\prime}$ and $5^{\prime}$ ends, the combination of circRNA with microRNA does not lead to its degradation. Therefore, circRNA can immobilize microRNA by "adsorption" similar to sponges, reducing target mRNA degradation and promoting its expression. ${ }^{28}$

Studies using antisense to the cerebellar degradationrelated protein I transcript (CDR1as) and cir-Sry ${ }^{16}$ present the strongest evidence that circRNA regulates gene expression by acting as a miRNA sponge. CDR1as, also known as circular RNA sponge for miR-7 (ciRS-7), contains more than 70 conserved miR-7 binding sites, which can play a powerful role as a miR-7 sponge. CDR1 as can bind to miR-7, resulting in decreased miR-7 activity and upregulated expression of its targeted mRNA. ${ }^{10,31}$ The testicular-specific Sry gene can transcribe an ecircRNA (cir-Sry) with sponge activity towards miR-138. ${ }^{32}$ cir-Sry contains 16 miR-138 binding sites that act as a sponge to inhibit miR-138 activity. cir-Sry is associated with the occurrence and development of malignant tumors, such as cholangiocarcinoma and colon, ovarian, and pulmonary cancer. ${ }^{33}$ These circRNA-miRNA-mRNA interaction networks regulate the occurrence and development of different diseases, including atherosclerosis, ${ }^{34}$ Parkinson's disease, ${ }^{28,35}$ Alzheimer's disease, ${ }^{36,37}$ diabetes, ${ }^{38-40}$ and pulmonary fibrosis. ${ }^{41,42}$

In recent years, the regulatory role of circRNA in tumorigenesis and tumor development has gradually become a new research hotspot. Liu et $\mathrm{al}^{43}$ found that Circ-SerPINE2 promoted cell proliferation and cell cycle progression and inhibited cell apoptosis in vitro by sponging miR-375 and regulating YWHAZ expression. In vivo, tumor growth was inhibited by increasing miR-375 expression and decreasing YWHAZ expression, suggesting that the Circ-SerPINE2/miR-375/YWHAZ axis may provide new therapeutic targets for gastric cancer. ${ }^{43}$ Two other studies proposed that regulatory networks, such as hsa_circ_0006215/miR-378a-3p/SERPINA4 and circBFAR/miR-34b-5p/MET/Akt, participate in the progression of pancreatic cancer. ${ }^{44,45}$

\section{CircRNA as a Protein Sponge}

In addition to being a miRNA sponge, circRNA can be a sponge for some proteins, thereby affecting their functional expression. circRNA can act as a sponge for RNA binding protein (RBP), forming the RNA-protein complex (RPC). ${ }^{46}$ $\mathrm{RBP}$ is involved in gene transcription and translation. Different circRNAs can indirectly regulate post-transcriptional processes by interacting with RBP, which affects the transcription of their parental genes (ie, the genes from which the circRNAs arise) and subsequent protein expression. CircRNA also plays an important role in the development of tumors and other diseases. ${ }^{47,48}$ For example, the circRNA MBL/MBNL1 contains a conserved binding site for muscleblind protein (MBL) ${ }^{23}$ Circ-PABPN1 can bind to HuR to prevent it from binding to PABPN1 mRNA, reducing the translation of this mRNA. ${ }^{49}$ CircEIF3J and CircPIAP2 can interact with the U1 snRNP and promote the transcription of their parental genes. ${ }^{18}$

Some circRNAs located in the cytoplasm can be protein sponges that directly regulate protein function. ${ }^{50} \mathrm{Du}$ et $\mathrm{al}^{51}$ found that circRNA-Foxo3 can prevent cell cycle progression by forming a complex with $\mathrm{p} 21$ and cyclin-dependent kinase 2 (CDK2). In another study, the same team found that Circ-Foxo3 was highly expressed in the cardiac specimens from elderly patients and mice and interacted with the antisenescence protein ID-1, transcription factor E2F1, and the 
anti-stress proteins FAK and HIF $1 .{ }^{52}$ These interactions retained the proteins in the cytoplasm, preventing them from exerting anti-senescence and anti-stress effects and causing intensified cell senescence.

In contrast to the antagonistic action of circRNA and proteins mentioned above, some circRNAs act synergistically with specific proteins potentially involved in regulating circRNA biogenesis under extracellular stimulation. For instance, a synergistic effect was observed between circRNAs and immune factor NF90/NF110 in promoting the immune response to virus infection. ${ }^{53} \mathrm{NF90/NF110,} \mathrm{which} \mathrm{is}$ generated from ILF3, is a dsRNA binding protein that binds the pairing bases of complementary sequences of introns juxtaposing the circRNA-forming exons, promoting the synthesis of circRNA in the nuclei. Furthermore, NF90/NF110 associates with circRNAs to form circ-RNP complexes, which are sequestered under non-infectious conditions. During a viral infection, NF90/NF110 is exported from the nucleus into the cytoplasm, resulting in the reduced expression of circRNA. Meanwhile, NF90/NF110 is released by the circRNP-complex and allowed to bind viral mRNA, participating in the anti-viral immune response.

\section{CircRNA as a Translation Template}

Several recent studies have shown that circRNA containing AUG sites and open reading frames (ORFs) can be driven by an internal ribosome entry site (IRES) and translated into polypeptides to perform biological functions. ${ }^{54,55}$ This discovery changed the previous misconception that circRNA could not play a coding role. In addition, the initiation of circRNA translation can be driven by chemical modifications, such as the m6A (N6-methyladenosine) modification. ${ }^{56}$ For instance, circ-ZNF609 binds to ribosomes through IRES and is translated into protein ZNF609 to regulate myoblast proliferation. ${ }^{57}$ Similarly, circ-FBXW7 can be directly translated into functional protein FBXW7-185aa, which shortens the half-life of c-Myc by antagonizing c-Myc stabilization induced by USP 28 and inhibits the proliferation of malignant glioma cells and the acceleration of cell cycle. ${ }^{58}$

\section{Circular RNA and Pancreatic Cancer Circular RNA and Development of Pancreatic Cancer CircRNA-Mediated miRNA Sponge Effect and Pancreatic Cancer}

CircRNA contains miRNA response elements (MREs) that can act as a miRNA sponge and compete with endogenous RNA to inhibit miRNA function, leading to the indirect upregulation of target gene expression levels. The circRNA-miRNA-mRNA signaling interaction network also plays an important regulatory role in the occurrence and development of pancreatic cancer. ${ }^{59}$ In particular, miRNA functions in cell proliferation, differentiation, apoptosis, and other activities. Similarly, to protooncogenes or tumor suppressor genes, it is also involved in the occurrence, development, invasion, and metastasis of malignant tumors. ${ }^{60}$ Therefore, circRNA can indirectly regulate the occurrence, proliferation, apoptosis, invasion, and metastasis of pancreatic cancer through its role as a miRNA sponge and even affect drug resistance and sensitivity.

With the advancements in sequencing technology, the circRNA expression profiles in pancreatic cancer tissue have been compared with normal pancreatic tissue by gene-chip technology. Multiple abnormalities in circRNA expression have been observed in pancreatic cancer tissue ${ }^{61-63}$ More than 20 circRNAs have been related to pancreatic cancer, and their biological functions have been verified by rigorous experiments (Table 1). Most of these studies focused on the miRNA sponge role of circRNA and the circRNA-miRNA-mRNA regulatory network. In these studies, the differentially expressed circRNA, miRNA, and mRNA molecules and the possible binding sites within them were identified through gene sequencing of pancreatic cancer tissue and paired para-carcinoma tissue to establish the hypothesis of the circRNAmiRNA-mRNA interaction network in this disease. The functions of these three types of RNAs were verified by in vitro cellular experiments and tumor-bearing models in vivo. Finally, the interaction between these three types of RNA and their corresponding signal transduction pathways were confirmed. By analyzing the clinical and pathological data of pancreatic cancer patients, researchers have established an association between differential circRNA expression and tumor biological behavior or patient prognosis.

As shown in Table 1, most of the biologically validated circRNAs are upregulated in pancreatic cancer tissues and cells, which is consistent with the status of circRNAs in other tumors. The first circRNA found to be upregulated and functionally validated in pancreatic cancer was circRNA_100782. ${ }^{64}$ Researchers sequenced the circRNAs from six pairs of pancreatic cancer and normal pancreatic tissues. circRNA_100782 displayed the most significant difference in expression. MicroRNA and target genes located downstream of circRNA_100782 were predicted 
Table I CircRNAs in Pancreatic Cancer and Their Molecular Mechanisms as miRNA Sponge

\begin{tabular}{|c|c|c|c|c|c|}
\hline CircRNA & Year & Expression & MiRNA Sponge & Signal Pathway & Ref. \\
\hline circRNA_I00782 & 2017 & Upregulated & miR-I24 & IL6/STAT3 & {$[64]$} \\
\hline hsa_circ_0006215 & 2018 & Upregulated & miR-378a-3p & SERPINA4 & {$[44]$} \\
\hline hsa_circ_0000977 & $\begin{array}{l}2018 \\
2019\end{array}$ & $\begin{array}{l}\text { Upregulated } \\
\text { Upregulated }\end{array}$ & $\begin{array}{l}\text { miR-874-3p } \\
\text { miR-I53 }\end{array}$ & $\begin{array}{l}\text { PLKI } \\
\text { HIFIA/ADAMIO }\end{array}$ & $\begin{array}{l}{[67]} \\
{[68]}\end{array}$ \\
\hline hsa_circ_0001649 & 2018 & Downregulated & - & - & [7I] \\
\hline circ-IARS & 2018 & Upregulated & miR-I22 & RhoA/F-actin/ZO-I & [69] \\
\hline hsa_circ_0099999 (circZMYM2) & 2018 & Upregulated & miR-335-5p & JMJD2C & {$[92]$} \\
\hline hsa_circ_0036627 (circ-PDE8A) & 2018 & Upregulated & miR-338 & MACC/MET/ERK & [93] \\
\hline hsa_circ_0007334 & 2019 & Upregulated & $\begin{array}{l}\text { miR-144-3p } \\
\text { miR-577 }\end{array}$ & $\begin{array}{l}\text { MMP7 } \\
\text { COLIAI }\end{array}$ & [94] \\
\hline circ_0030235 & 2019 & Upregulated & miR-I253/miR-I294 & - & {$[80]$} \\
\hline circ-ASH2L & 2019 & Upregulated & $\operatorname{miR}-34 a$ & Notchl & {$[95]$} \\
\hline hsa_circ_0005397 (circRHOTI) & $\begin{array}{l}2019 \\
2020\end{array}$ & $\begin{array}{l}\text { Upregulated } \\
\text { Upregulated }\end{array}$ & $\begin{array}{l}\mathrm{miR}-26 \mathrm{~b} / \mathrm{miR}-\mathrm{I} 25 \mathrm{a} / \mathrm{miR}-330 / \mathrm{miR}-382 \\
\mathrm{miR}-125 \mathrm{a}-3 \mathrm{p}\end{array}$ & $\begin{array}{l}- \\
\text { E2F3 }\end{array}$ & $\begin{array}{l}{[96]} \\
{[97]}\end{array}$ \\
\hline circ-ADAM9 & 2019 & Upregulated & miR-2I7 & PRSS3/ERK/VEGF & [98] \\
\hline ciRS-7 (Cdrlas) & $\begin{array}{l}2019 \\
2021\end{array}$ & $\begin{array}{l}\text { Upregulated } \\
\text { Upregulated }\end{array}$ & $\begin{array}{l}\text { miR-7 } \\
\text { miR-432-5p }\end{array}$ & $\begin{array}{l}\text { EGFR/STAT3 } \\
\text { E2F3 }\end{array}$ & $\begin{array}{l}{[66]} \\
{[99]}\end{array}$ \\
\hline circ_0007534 & 2019 & Upregulated & $\mathrm{miR}-625 / \mathrm{miR}-892 \mathrm{~b}$ & - & {$[8 \mid]$} \\
\hline hsa_circ_0006988 (circ-LDLRAD3) & 2019 & Upregulated & miR-137-3p & PTN & {$[100]$} \\
\hline circFOXK2 & 2020 & Upregulated & miR-942 & ANKI/GDNF/PAX6 & [73] \\
\hline circHIPK3 & 2020 & Upregulated & miR-330-5p & RASSFI & {$[90]$} \\
\hline hsa_circ_001653 & 2020 & Upregulated & miR-37 & HOXC6 & {$[101]$} \\
\hline hsa_circ_0086375 (circNFIBI) & 2020 & Downregulated & miR-486-5p & PIK3RI/VEGF-C & {$[70]$} \\
\hline hsa_circ_0009065 (circBFAR) & 2020 & Upregulated & miR-34b-5p & MET/PI3K/Akt & {$[45]$} \\
\hline hsa_circ_0013912 & 2020 & Upregulated & miR-7-5p & - & [102] \\
\hline hsa_circRNA_00I587 (circRNA0000979) & 2020 & Downregulated & miR-223 & SLC4A4 & {$[72]$} \\
\hline hsa_circ_0066I47 (circSFMBTI) & 2020 & Upregulated & miR-330-5p & PAKI & [103] \\
\hline CircRNA_000864 & 2020 & Downregulated & miR-36I-3p & BTG2 & {$[104]$} \\
\hline circEIF6 & 2021 & Upregulated & miR-557 & /SLC7AII/PI3K/Akt & {$[105]$} \\
\hline circNEIL3 & 2021 & Upregulated & miR-432-5p & ADARI & [106] \\
\hline hsa_circ_007I 036 & 2021 & Upregulated & miR-489 & - & [107] \\
\hline
\end{tabular}

by bioinformatics analysis tools, and their biological functions and interactions with each other were confirmed by in vitro cell experiments. Further investigation showed that circRNA_100782 is a miR-124 sponge that regulates the proliferation of pancreatic cancer cells through the IL6-
STAT3 pathway. It affects the biological behavior of pancreatic cancer and plays a role in promoting pancreatic cancer. Subsequently, STAT3 upregulation was shown to mediate the immunologic escape of tumors. ${ }^{65}$ Accordingly, circRNA_100782 might also be involved in 
tumor immunity in pancreatic cancer by a mechanism that requires further investigation. Because this study was only conducted to verify and compare the expression of circRNA_100782 and miR-124 using two different pancreatic cancer cell lines (ie, BxPC3 and HPDE) ${ }^{64}$ the results were poorly representative of the disease. Furthermore, because this comparison was not performed in parallel in pancreatic cancer tissues, the reliability of the verification was not established. Since those studies were performed, the procedures and methods in similar studies have developed and improved. Researchers have discovered that numerous circRNAs act as miRNA sponges in pancreatic cancer and have constructed dozens of circRNA-miRNA-mRNA regulatory networks. Analogously, the mechanism of ciRS-7 and miR-7 in the progression of pancreatic cancer involves the targeting of miR-7 by ciRS-7 to regulate the EGFR/STAT3 signal pathway, which promotes the expression of these two factors. ${ }^{66}$

CircRNA studies have gradually expanded the sample capacity of pancreatic cancer tissues and collected clinical patient data for statistical analysis, linking the differentially expressed RNA molecules with the clinicopathology and prognosis of the pancreatic cancer patients. For instance, the expression of circBFAR (hsa_circ_0009065) was upregulated in 208 patients with pancreatic ductal adenocarcinoma (PDAC), and ectopic circBFAR expression was positively correlated with the stage of lymph node metastasis (TNM) related to poor prognosis for PDAC patients. ${ }^{45}$ circBFAR upregulates the expression of mesenchymal-epithelial transition factor (MET) by sponging miR-34b-5p and activating the MET/PI3K/Akt signaling pathway, which ultimately promotes PDAC proliferation and metastasis. This study revealed that MET inhibitors could reverse the carcinogenic effect induced by circBFAR overexpression in vivo, indicating that circBFAR could be used as a potential prognostic indicator and therapeutic target for PDAC. Similarly, hsa_circ_0000977 is overexpressed in pancreatic cancer tissues and is closely associated with negative clinical characteristics and poor prognosis. ${ }^{67}$ Therefore, hsa_circ_0000977 overexpression may be a useful biomarker for the prognosis of PDAC. Additional studies have shown that the hsa_circ_0000977/miR-874-3P/PLK1 axis plays an important role in regulating pancreatic cancer cell proliferation. They also found that the silencing of this circRNA inhibits the growth of pancreatic cancer cells both in vivo and in vitro, suggesting a novel therapeutic strategy against pancreatic cancer.
Unlike the above studies that directly measured circRNA expression in pancreatic cancer cell lines, some studies have focused on the variations of circRNA expression in pancreatic cancer cells under specific cultural conditions (eg, hypoxia) to infer the molecular mechanism involved in the proliferation and metastasis of pancreatic cancer. Ou et $\mathrm{al}^{68}$ found that a hypoxic immune microenvironment significantly enhanced hsa_circ_0000977 expression in advanced pancreatic cancer. Through competitive binding with miR153, hsa_circ_0000977 antagonizes miR-153-mediated inhibition of HIF1A and ADAM10, leading to their increased expression and allowing the HIF1A-mediated immune escape by pancreatic cancer cells. ${ }^{68}$ The silencing of hsa_circ_0000977 significantly enhances the cytotoxic effect of natural killer cells on pancreatic cancer. Thus, targeting hsa_circ_0000977 is expected to play an immunosensitizing role in the treatment or prevention of pancreatic cancer.

In addition to pancreatic cancer tissues and cells, circRNA is also widely found in plasma exosomes from patients with pancreatic cancer and participates in intercellular signal transduction. For example, Circ-IARS is overexpressed in tissues and plasma exocrine bodies from pancreatic cancer patients and is positively correlated with tumor metastasis but negatively correlated with patient survival. ${ }^{69}$ Additional research showed that circ-IARS from pancreatic cancer patient plasma exosomes could upregulate RhoA activity and F-actin expression and reduce ZO-1 expression by binding with miR-122 upon entering human umbilical vein endothelial cells (HUVECs). These circ-IARS-induced changes enhanced the permeability of the endothelial monolayer, promoting invasion and metastasis. These data also suggest that the presence of circRNA in exosomes may serve as a crucial indicator for early diagnosis and prognosis for PDAC.

Unlike the differentially expressed circRNAs mentioned above, the expression levels of some circRNAs are downregulated in pancreatic cancer, which diminishes their inhibitory effect on downstream carcinogenic miRNAs, leading to the malignant biological behaviors of tumors and poor patient prognosis. Artificial overexpression or exogenous administration of these circRNAs may inhibit the proliferation of pancreatic cancer cells and pancreatic cancer development. For instance, hsa_circ_0086375 (circNFIB1) is downregulated in PDAC and negatively correlated with lymph node metastasis. ${ }^{70}$ Conversely, knockout of this circRNA gene promotes lymphangiogenesis and lymph node metastasis of PDAC. circNFIB1 acts as a miR-486-5p sponge to partially reverse the carcinogenic effect of this miRNA. It upregulates PIK3R1expression and downregulates VEGF-C expression by 
inhibiting the PI3K/Akt pathway, which subsequently inhibits the generation of lymphatic vessels and PDAC lymph node metastasis. Similarly, hsa_circ_0001649 ${ }^{71}$ and hsa_circ_001587 $7^{72}$ are downregulated in pancreatic cancer. hsa_circ_0001649 is downregulated in PDAC tissues and cells, which is generally accompanied by the advanced tumor stage and a reduction in histological grade. Exogenous hsa circ_0001649 administration inhibits the proliferation of pancreatic cancer cells and induces apoptosis, suggesting that hsa_circ_0001649 could be used as a potential antitumor agent. $^{71}$ hsa_circRNA_001587 inhibits the migration, invasion, angiogenesis, and tumorigenesis of pancreatic cancer cells by impairing miR-223-mediated inhibition of SLC4A4. ${ }^{72}$ Collectively, these circRNAs appear to inhibit the progression of pancreatic cancer.

\section{Other Effects of circRNA and Pancreatic Cancer}

In addition to acting as a miRNA sponge in pancreatic cancer, other circRNA functions have been demonstrated in this disease, including acting as an RNA-binding protein sponge or a regulator of parental gene expression. Wong et $\mathrm{al}^{73}$ found that circFOXK2 was significantly upregulated in PDAC cells, including $63 \%(53 / 84)$ of primary tumors by circRNA sequencing. This circRNA promoted the growth, invasion, and liver metastasis of PDAC cells. CircFOXK2 contains multiple miRNA binding sites and acts as a sponge for miR-942 to promote the expression of ANK1, GDNF, and PAX6. In addition, a variety of proteins interacting with CircFOXK2 were identified by mass spectrometry. Among these proteins, the interaction between CircFOXK2 and YBX1 and hnRNPK enhanced the expression of the oncogenes NUF2 and PDXK and promoted the invasion of PDAC cells. As an RNA-binding protein, YBX1 regulates the transcription, processing, and translation of mRNA. Moreover, YBX1 is upregulated in various malignant tumors, including pancreatic cancer, and is involved in tumor cell invasion and chemoresistance. ${ }^{74-76}$ Furthermore, Ye et $\mathrm{al}^{77}$ found that downregulation of hsa_circ_0000069 markedly suppressed the expression of its parental gene, SCL/TAL1 interrupting locus (STIL) and inhibited the proliferation, migration, and invasion of pancreatic cancer cells. These studies suggest that circRNA can be more powerful than just a miRNA sponge in pancreatic cancer.

\section{Circular RNA in Clinical Diagnosis and Treatment of Pancreatic Cancer CircRNA as a New Biomarker for Pancreatic Cancer} CircRNA expression is tissue-, time-, and disease-specific with high expression levels and stability in cells, tissues, and body fluids. ${ }^{78,79}$ Thus, circRNA is a potential biomarker for disease diagnosis. Several studies (Table 1) have shown that various circRNAs are differentially expressed in pancreatic cancer tissues compared to normal tissues. These different expression profiles are related to the biological behavior of pancreatic cancer and patient prognosis, suggesting they can be used as new biomarkers to diagnose this disease. ${ }^{64,71,80-82}$ hsa_circ_0006988 (circLDLRAD3) expression is significantly higher in pancreatic cancer tissues and plasma than in control samples. In addition, upregulated circ-LDLRAD3 expression is significantly correlated with the main clinicopathological factors of pancreatic cancer patients. ${ }^{82}$ The expression of circLDLRAD3 in pancreatic cancer tissues is also closely associated with vascular invasion and lymphatic metastasis. In plasma, its expression is correlated with CA19-9 levels, TNM stage, vascular invasion, and lymphatic metastasis. Therefore, circ-LDLRAD3 could be used as a biomarker for the early diagnosis of this disease. Moreover, high sensitivity and specificity were observed for circ-LDLRAD3 combined with CA19-9 in aiding early diagnosis. Moreover, the upregulation of hsa_circ_0007534 ${ }^{81}$ and hsa_circ_0030235 $5^{80}$ suggest poor prognosis for PDAC patients. Furthermore, the upregulation of hsa_circ_0007534 is also indicative of lymph node infiltration, ${ }^{81}$ while the high expression of hsa_circ_0030235 is associated with positive lymph nodes and higher tumor stage. ${ }^{80}$ Finally, hsa_circ_0001649 expression in PDAC cells and tissues is significantly downregulated. Its low expression suggests irreversible differentiation and staging, while its high expression predicts a higher overall survival rate. ${ }^{71}$

\section{CircRNA in Targeted Therapy and Drug Resistance of Pancreatic Cancer}

CircRNA performs complex, diverse functions and plays a role in carcinogenic signaling pathways involving different molecules (eg, miRNAs, lncRNAs, RNA-binding proteins, and transcription factors), which can influence the occurrence and development of various tumors. Because targeting circRNA would not only regulate multiple oncogenes simultaneously but also miRNA, it would be a superior targeted therapy compared to targeting a single miRNA or oncogene. The basic strategy for targeted therapy against overexpressed circRNA in malignant tumors is to suppress the expression of the tumor-promoting circRNA through gene knockout, antisense oligonucleotide RNA, small interfering RNA (siRNA), or artificial tumor suppressor circRNAs. ${ }^{83}$ siRNAs could 
combine the specific reverse splicing sites of circRNA with the characteristic sequences in precursor mRNAs (eg, upstream acceptor, downstream donor, and Alu elements), resulting in the failed formation of the RNA lariat. ${ }^{84}$ In addition, CRISPR/ Cas9 knockout of the circRNA expression gene, ${ }^{85}$ exogenous administration of tumor-suppressing circRNAs, and the synthesis of circRNA inhibiting oncogenic miRNA ${ }^{86}$ are also of clinical value. For the downregulation of circRNAs, the expression levels of tumor-suppressing circRNAs can be restored by cloning circRNA and regulating the lateral regions. $^{84}$

As shown in Table 1, silencing of some highly expressed circRNAs in pancreatic cancer can enhance the functions of downstream miRNAs, which inhibit the carcinogenic effect of the miRNA target genes and, thus, the progression of pancreatic cancer. These data suggest that some circRNAs may be potential therapeutic targets for PDAC treatment. In addition, ten differentially expressed circRNAs, 12 miRNAs, and 118 mRNA interacting with each other were identified by a combination of gene chip analysis and bioinformatics, along with their possible mechanisms. This analysis established circRNAmiRNA-mRNA networks in the molecular mechanism of pancreatic cancer. ${ }^{87}$ These 118 mRNAs were used to construct a protein-protein interaction network. This network identified four hub genes - CdH1, SERPINE1, IRS1, and FYN. Subsequently, CMap analysis identified three bioactive compounds (Celastrol, 5109870, and MMG -132) that could be used to treat PDAC.

Furthermore, circRNAs may also be involved in the chemotherapeutic resistance of pancreatic cancer. A comparison of circRNA expression profiles between drug-resistant pancreatic cancer cell lines and the drug-sensitive parental cells identified differentially expressed circRNAs. The potential roles of these differentially expressed circRNAs in drug resistance were investigated. For example, circRNA expression profiles in gemcitabine-resistant Sw1990 pancreatic cancer cell lines and the gemcitabine-sensitive parental Sw1990 cell line demonstrated that circRNA_101672, circRNA_004077, and circRNA_003251 were significantly upregulated in the drug-resistant cell lines, and circRNA_101543, circRNA_102747, and circRNA_000926 were markedly downregulated. ${ }^{88}$ These dysregulated circRNAs may act as miRNA sponges, thereby affecting the MAPK and mTOR signaling pathways and mediating chemotherapeutic resistance. Similarly, Shao et al ${ }^{89}$ compared differences in the circRNA expression profiles between gemcitabine-resistant PANC-1-GR cells and parental PANC-1 cells by RNA sequencing and determined that two circRNAs (located at chr14:101402109-101464448+ and chr4:52729603-$52780244+$ ) were differentially expressed. They also found that silencing these two circRNAs restored the susceptibility of PANC-1-GR cells to gemcitabine, while their overexpression reduced the sensitivity, suggesting that they may be novel biomarkers and potential therapeutic targets for gemcitabineresistant pancreatic cancer. Liu et $\mathrm{al}^{90}$ found that circHIPK3 promoted gemcitabine resistance in pancreatic cancer cells by targeting RASSF1 via the sponging of miR-330-5p. Therefore, circHIPK3 may also be a biomarker for drug resistance in pancreatic cancer and a prognostic indicator. ${ }^{91}$

\section{Summary and Prospect}

In this review, the classification, biogenesis, and functions of circRNA were briefly introduced, and the current research on the molecular mechanism of circRNA and its potential use in the clinical diagnosis and treatment of pancreatic cancer was reviewed in detail. In recent years, circRNA has become a new research hotspot. With the increasing progress in technology and methods (eg, gene chip and sequencing), circRNA databases are being enriched and improved. CircRNA functions in transcriptional regulation and polypeptide translation function and as a microRNA sponge. Its associations with some diseases, especially various types of tumors, have been confirmed. Studies have suggested that most circRNAs play an endogenous competitive role as miRNA sponges in tumors, while some circRNAs can directly bind to proteins and act as sponges or synergistic factors to regulate tumor growth, invasion, and migration, all of which can affect the prognosis of patients. Compared to traditional linear RNAs and other RNA molecule families (eg, lncRNA and miRNA), circRNA is time- and tissue-specific and widely expressed in various tissues, cells, and body fluids, with strong structural stability. These characteristics make it possible for circRNA to be an effective biomarker in the early diagnosis, targeted therapy, and prognosis evaluation of the diseases.

A number of studies have verified that the expression profile of circRNA in pancreatic cancer tissues is significantly different from that in normal tissues. These differentially expressed circRNAs can regulate the expression of downstream target genes by binding to specific miRNAs. The circRNA-miRNA-mRNA signal axis can regulate the biological behaviors (eg, invasion, metastasis, immune escape, and chemoresistance) of pancreatic cancer. Further research of these signaling pathways underlies our search for diagnostic markers and therapeutic targets for this disease. 
The circRNA research into the diagnosis and treatment of pancreatic cancer is still in an early stage, and the following directions can be explored with future research. First, current circRNA research in pancreatic cancer has been mainly limited to the function of circRNA as a miRNA sponge. However, only a few circRNAs act as miRNA sponges. Indeed, circRNAs also serve as protein sponges, transcriptional regulators, and peptide translation templates and may also play other roles. Therefore, the next stage of circRNA research should focus on these other circRNA functions and their molecular mechanisms underlying pancreatic cancer. Secondly, there is still an absence of convincing clinical studies and animal experiments in the field of circRNA research in pancreatic cancer to determine the potential of circRNAs in clinical therapy. The next research focus in this area will be on how to transform the reported circRNA data related to pancreatic cancer into targeted therapeutic drugs for the treatment of this disease. In addition, some scholars have found that circRNA can participate in intercellular signal transduction using exosomes as a medium; however, the specific mechanism has not been defined. It is believed that with the continuous progress in experimental technology and research, circRNA will eventually play an important role in the early diagnosis, targeted therapy, and prognosis improvement of pancreatic cancer.

\section{Acknowledgments}

This study was supported by the National Natural Science Foundation of China (Grant No. 81900601), the Natural Science Foundation of Liaoning Province (Grant No. 20180530014), University innovation team and innovative talent support program of Liaoning Province (Grant No. LR2019073), and Outstanding Scientific Fund of Shengjing Hospital (Grant No. 201701).

\section{Disclosure}

Dr Nan Ge reports grants from Natural Science Foundation of Liaoning Province (Grant No. 20180530014), during the conduct of the study; Dr Jintao Guo report grants from National Natural Science Foundation of China (Grant No. 81900601), during the conduct of the study.

Dr Siyu Sun reports grants from University innovation team and innovative talent support program of Liaoning Province (Grant No. LR2019073), from Outstanding Scientific Fund of Shengjing Hospital (Grant No. 201701), during the conduct of the study. The authors report no conflicts of interest in this work.

\section{References}

1. Kristensen LS, Andersen MS, Stagsted LVW, Ebbesen KK, Hansen TB, Kjems J. The biogenesis, biology and characterization of circular RNAs. Nat Rev Genet. 2019;20(11):675-691. doi:10.1038/s41576-019-0158-7

2. Sanger HL, Klotz G, Riesner D, Gross HJ, Kleinschmidt AK. Viroids are single-stranded covalently closed circular RNA molecules existing as highly base-paired rod-like structures. Proc Natl Acad Sci U S A. 1976;73(11):3852-3856. doi:10.1073/ pnas.73.11.3852

3. Hsu MT, Coca-Prados M. Electron microscopic evidence for the circular form of RNA in the cytoplasm of eukaryotic cells. Nature. 1979;280(5720):339-340. doi:10.1038/280339a0

4. Arnberg AC, Van Ommen GJ, Grivell LA, Van Bruggen EF, Borst P. Some yeast mitochondrial RNAs are circular. Cell. 1980;19(2):313-319. doi:10.1016/0092-8674(80)90505-X

5. Wang PL, Bao Y, Yee MC, et al. Circular RNA is expressed across the eukaryotic tree of life. PLoS One. 2014;9(6):e90859. doi:10.1371/journal.pone.0090859

6. Cocquerelle C, Mascrez B, Hétuin D, Bailleul B. Mis-splicing yields circular RNA molecules. FASEB J. 1993;7(1):155-160. doi:10.1096/fasebj.7.1.7678559

7. Zhang XO, Wang $\mathrm{HB}$, Zhang Y, Lu X, Chen LL, Yang L. Complementary sequence-mediated exon circularization. Cell. 2014;159(1):134-147. doi:10.1016/j.cell.2014.09.001

8. Suzuki H, Tsukahara T. A view of pre-mRNA splicing from RNase R resistant RNAs. Int J Mol Sci. 2014;15(6):9331-9342. doi:10.3390/ijms15069331

9. Venø MT, Hansen TB, Venø ST, et al. Spatio-temporal regulation of circular RNA expression during porcine embryonic brain development. Genome Biol. 2015;16:245. doi:10.1186/s13059015-0801-3

10. Guo JU, Agarwal V, Guo H, Bartel DP. Expanded identification and characterization of mammalian circular RNAs. Genome Biol. 2014;15(7):409. doi:10.1186/s13059-014-0409-Z

11. Jeck WR, Sharpless NE. Detecting and characterizing circular RNAs. Nat Biotechnol. 2014;32(5):453-461. doi:10.1038/ nbt. 2890

12. Sun XD, Huan C, Sun DW, Lv GY. Prognostic and clinicopathological significance of circular RNA circ-ITCH expression in cancer patients: a meta-analysis. Biomed Res Int. 2021;2021:8828299. doi:10.1155/2021/8828299

13. Zuo L, Zhang $\mathrm{L}, \mathrm{Zu} \mathrm{J}$, et al. Circulating circular RNAs as biomarkers for the diagnosis and prediction of outcomes in acute ischemic stroke. Stroke. 2020;51(1):319-323. doi:10.1161/ STROKEAHA.119.027348

14. Siegel RL, Miller KD, Jemal A. Cancer statistics, 2020. $C A$ Cancer J Clin. 2020;70(1):7-30. doi:10.3322/caac.21590

15. Rahib L, Smith BD, Aizenberg R, Rosenzweig AB, Fleshman JM, Matrisian LM. Projecting cancer incidence and deaths to 2030: the unexpected burden of thyroid, liver, and pancreas cancers in the United States. Cancer Res. 2014;74(11):2913-2921. doi:10.1158/0008-5472.CAN-14-0155

16. Mumtaz PT, Taban Q, Dar MA, et al. Deep Insights in circular RNAs: from biogenesis to therapeutics. Biol Proced Online. 2020;22:10. doi:10.1186/s12575-020-00122-8

17. Zhang Y, Zhang XO, Chen T, et al. Circular intronic long noncoding RNAs. Mol Cell. 2013;51(6):792-806. doi:10.1016/j. molcel.2013.08.017

18. Li Z, Huang C, Bao C, et al. Exon-intron circular RNAs regulate transcription in the nucleus. Nat Struct Mol Biol. 2015;22 (3):256-264. doi:10.1038/nsmb.2959

19. Lu Z, Filonov GS, Noto JJ, et al. Metazoan tRNA introns generate stable circular RNAs in vivo. RNA. 2015;21(9):1554-1565. doi:10.1261/rna.052944.115 
20. Noto JJ, Schmidt CA, Matera AG. Engineering and expressing circular RNAs via tRNA splicing. RNA Biol. 2017;14 (8):978-984. doi:10.1080/15476286.2017.1317911

21. Patop IL, Wüst S, Kadener S. Past, present, and future of circRNAs. EMBO J. 2019;38(16):e100836. doi:10.15252/ embj.2018100836

22. Jeck WR, Sorrentino JA, Wang K, et al. Circular RNAs are abundant, conserved, and associated with ALU repeats. RNA. 2013;19(2):141-157. doi:10.1261/rna.035667.112

23. Ashwal-Fluss R, Meyer M, Pamudurti NR, et al. circRNA biogenesis competes with pre-mRNA splicing. Mol Cell. 2014;56 (1):55-66. doi:10.1016/j.molcel.2014.08.019

24. Kameyama T, Suzuki H, Mayeda A. Re-splicing of mature mRNA in cancer cells promotes activation of distant weak alternative splice sites. Nucleic Acids Res. 2012;40(16):7896-7906. doi:10.1093/nar/gks520

25. Qu S, Yang X, Li X, et al. Circular RNA: a new star of noncoding RNAs. Cancer Lett. 2015;365(2):141-148. doi:10.1016/j. canlet.2015.06.003

26. Abelson J, Trotta CR, Li H. tRNA splicing. J Biol Chem. 1998;273(21):12685-12688. doi:10.1074/jbc.273.21.12685

27. Schmidt CA, Giusto JD, Bao A, Hopper AK, Matera AG. Molecular determinants of metazoan tricRNA biogenesis. Nucleic Acids Res. 2019;47(12):6452-6465. doi:10.1093/nar/ gkz311

28. Hansen TB, Jensen TI, Clausen BH, et al. Natural RNA circles function as efficient microRNA sponges. Nature. 2013;495 (7441):384-388. doi:10.1038/nature11993

29. Memczak S, Jens M, Elefsinioti A, et al. Circular RNAs are a large class of animal RNAs with regulatory potency. Nature. 2013;495(7441):333-338. doi:10.1038/nature 11928

30. Wilusz JE. A $360^{\circ}$ view of circular RNAs: from biogenesis to functions. Wiley Interdiscip Rev RNA. 2018;9(4):e1478. doi:10.1002/wrna.1478

31. Peng L, Yuan XQ, Li GC. The emerging landscape of circular RNA ciRS-7 in cancer (Review). Oncol Rep. 2015;33 (6):2669-2674. doi:10.3892/or.2015.3904

32. Capel B, Swain A, Nicolis S, et al. Circular transcripts of the testis-determining gene Sry in adult mouse testis. Cell. 1993;73 (5):1019-1030. doi:10.1016/0092-8674(93)90279-Y

33. Zhao ZJ, Shen J. Circular RNA participates in the carcinogenesis and the malignant behavior of cancer. RNA Biol. 2017;14 (5):514-521. doi:10.1080/15476286.2015.1122162

34. Wang G, Li Y, Liu Z, et al. Circular RNA circ_0124644 exacerbates the ox-LDL-induced endothelial injury in human vascular endothelial cells through regulating PAPP-A by acting as a sponge of miR-149-5p. Mol Cell Biochem. 2020;471(1-2):51-61. doi:10.1007/s11010-020-03764-0

35. Junn E, Lee KW, Jeong BS, Chan TW, Im JY, Mouradian MM. Repression of alpha-synuclein expression and toxicity by microRNA-7. Proc Natl Acad Sci U S A. 2009;106 (31):13052-13057. doi:10.1073/pnas.0906277106

36. Lukiw WJ. Circular RNA (circRNA) in Alzheimer's disease (AD). Front Genet. 2013;4:307. doi:10.3389/fgene.2013.00307

37. Zhao Y, Alexandrov PN, Jaber V, Lukiw WJ. Deficiency in the ubiquitin conjugating enzyme UBE2A in Alzheimer's disease (AD) is linked to deficits in a natural circular miRNA-7 sponge (circRNA; ciRS-7). Genes (Basel). 2016;7:12. doi:10.3390/ genes7120116

38. Yan Q, He X, Kuang G, Ou C. CircRNA cPWWP2A: an emerging player in diabetes mellitus. $J$ Cell Commun Signal. 2020;14 (3):351-353. doi:10.1007/s12079-020-00570-7

39. Wang Y, Liu J, Liu C, Naji A, Stoffers DA. MicroRNA-7 regulates the mTOR pathway and proliferation in adult pancreatic $\beta$-cells. Diabetes. 2013;62(3):887-895. doi:10.2337/ $\mathrm{db} 12-0451$
40. Xu H, Guo S, Li W, Yu P. The circular RNA Cdr1as, via miR-7 and its targets, regulates insulin transcription and secretion in islet cells. Sci Rep. 2015;5:12453. doi:10.1038/srep12453

41. Lu G, Zhang J, Liu X, et al. Regulatory network of two circRNAs and an miRNA with their targeted genes under astilbin treatment in pulmonary fibrosis. J Cell Mol Med. 2019;23(10):6720-6729. doi: $10.1111 /$ jcmm. 14550

42. Bachmayr-Heyda A, Reiner AT, Auer K, et al. Correlation of circular RNA abundance with proliferation-exemplified with colorectal and ovarian cancer, idiopathic lung fibrosis, and normal human tissues. Sci Rep. 2015;5:8057. doi:10.1038/srep08057

43. Liu J, Song S, Lin S, et al. Circ-SERPINE2 promotes the development of gastric carcinoma by sponging miR-375 and modulating YWHAZ. Cell Prolif. 2019;52(4):e12648. doi:10.1111/cpr.12648

44. Zhu P, Ge N, Liu D, et al. Preliminary investigation of the function of hsa_circ_0006215 in pancreatic cancer. Oncol Lett. 2018;16(1):603-611. doi:10.3892/ol.2018.8652

45. Guo X, Zhou Q, Su D, et al. Circular RNA circBFAR promotes the progression of pancreatic ductal adenocarcinoma via the miR-34b-5p/MET/Akt axis. Mol Cancer. 2020;19(1):83. doi:10.1186/s12943-020-01196-4

46. Zang J, Lu D, Xu A. The interaction of circRNAs and RNA binding proteins: an important part of circRNA maintenance and function. J Neurosci Res. 2020;98(1):87-97. doi:10.1002/jnr.24356

47. Tang Q, Hann SS. Biological roles and mechanisms of circular RNA in human cancers. Onco Targets Ther. 2020;13:2067-2092. doi:10.2147/OTT.S233672

48. Xu S, Zhou L, Ponnusamy M, et al. A comprehensive review of circRNA: from purification and identification to disease marker potential. PeerJ. 2018;6:e5503. doi:10.7717/peerj.5503

49. Abdelmohsen K, Panda AC, Munk R, et al. Identification of HuR target circular RNAs uncovers suppression of PABPN1 translation by CircPABPN1. RNA Biol. 2017;14(3):361-369. doi:10.1080/15476286.2017.1279788

50. Zhang Z, Yang T, Xiao J. Circular RNAs: promising biomarkers for human diseases. EBioMedicine. 2018;34:267-274. doi:10.1016/j.ebiom.2018.07.036

51. Du WW, Yang W, Liu E, Yang Z, Dhaliwal P, Yang BB. Foxo3 circular RNA retards cell cycle progression via forming ternary complexes with p21 and CDK2. Nucleic Acids Res. 2016;44 (6):2846-2858. doi:10.1093/nar/gkw027

52. Du WW, Yang W, Chen Y, et al. Foxo3 circular RNA promotes cardiac senescence by modulating multiple factors associated with stress and senescence responses. Eur Heart J. 2017;38 (18):1402-1412. doi:10.1093/eurheartj/ehw001

53. Li X, Liu CX, Xue W, et al. Coordinated circRNA biogenesis and function with NF90/NF110 in viral infection. Mol Cell. 2017;67 (2):214-227.e217. doi:10.1016/j.molcel.2017.05.023

54. Pamudurti NR, Bartok O, Jens M, et al. Translation of circRNAs. Mol Cell. 2017;66(1):9-21.e27. doi:10.1016/j.molcel.2017.02.021

55. Das A, Gorospe M, Panda AC. The coding potential of circRNAs. Aging (Albany NY). 2018;10(9):2228-2229. doi:10.18632/ aging. 101554

56. Yang Y, Fan X, Mao M, et al. Extensive translation of circular RNAs driven by N(6)-methyladenosine. Cell Res. 2017;27 (5):626-641. doi:10.1038/cr.2017.31

57. Legnini I, Di Timoteo G, Rossi F, et al. Circ-ZNF609 is a circular RNA that can be translated and functions in myogenesis. Mol Cell. 2017;66(1):22-37.e29. doi:10.1016/j.molcel.2017.02.017

58. Yang Y, Gao X, Zhang M, et al. Novel role of FBXW7 circular RNA in repressing glioma tumorigenesis. $J$ Natl Cancer Inst. 2018;110(3):304-315. doi:10.1093/jnci/djx166

59. Song W, Wang WJ, Fu T, Chen L, Miao DL. Integrated analysis of circular RNA-associated ceRNA network in pancreatic ductal adenocarcinoma. Oncol Lett. 2020;19(3):2175-2184. doi:10.3892/ol.2020.11306 
60. Vishnoi A, Rani S. MiRNA biogenesis and regulation of diseases: an overview. Methods Mol Biol. 2017;1509:1-10.

61. Li H, Hao X, Wang H, et al. Circular RNA expression profile of pancreatic ductal adenocarcinoma revealed by microarray. Cell Physiol Biochem. 2016;40(6):1334-1344. doi:10.1159/000453186

62. Guo S, Xu X, Ouyang Y, et al. Microarray expression profile analysis of circular RNAs in pancreatic cancer. Mol Med Rep. 2018;17(6):7661-7671. doi:10.3892/mmr.2018.8827

63. Zhang Q, Wang JY, Zhou SY, Yang SJ, Zhong SL. Circular RNA expression in pancreatic ductal adenocarcinoma. Oncol Lett. 2019;18(3):2923-2930. doi:10.3892/ol.2019.10624

64. Chen G, Shi Y, Zhang Y, Sun J. CircRNA_100782 regulates pancreatic carcinoma proliferation through the IL6-STAT3 pathway. Onco Targets Ther. 2017;10:5783-5794. doi:10.2147/OTT.S150678

65. Yang ZG, Awan FM, Du WW, et al. The circular RNA interacts with STAT3, increasing its nuclear translocation and wound repair by modulating Dnmt3a and miR-17 function. Mol Ther. 2017;25 (9):2062-2074. doi:10.1016/j.ymthe.2017.05.022

66. Liu L, Liu FB, Huang M, et al. Circular RNA ciRS-7 promotes the proliferation and metastasis of pancreatic cancer by regulating miR-7-mediated EGFR/STAT3 signaling pathway. Hepatobiliary Pancreat Dis Int. 2019;18(6):580-586. doi:10.1016/j. hbpd.2019.03.003

67. Huang WJ, Wang Y, Liu S, et al. Silencing circular RNA hsa_circ_0000977 suppresses pancreatic ductal adenocarcinoma progression by stimulating miR-874-3p and inhibiting PLK1 expression. Cancer Lett. 2018;422:70-80. doi:10.1016/j. canlet.2018.02.014

68. Ou ZL, Luo Z, Wei W, Liang S, Gao TL, Lu YB. Hypoxiainduced shedding of MICA and HIF1A-mediated immune escape of pancreatic cancer cells from NK cells: role of circ_0000977/ miR-153 axis. RNA Biol. 2019;16(11):1592-1603. doi:10.1080/ 15476286.2019.1649585

69. Li J, Li Z, Jiang P, et al. Circular RNA IARS (circ-IARS) secreted by pancreatic cancer cells and located within exosomes regulates endothelial monolayer permeability to promote tumor metastasis. $J$ Exp Clin Cancer Res. 2018;37(1):177. doi:10.1186/s13046-0180822-3

70. Kong Y, Li Y, Luo Y, et al. circNFIB1 inhibits lymphangiogenesis and lymphatic metastasis via the miR-486-5p/PIK3R1/VEGF-C axis in pancreatic cancer. Mol Cancer. 2020;19(1):82 doi:10.1186/s12943-020-01205-6

71. Jiang Y, Wang T, Yan L, Qu L. A novel prognostic biomarker for pancreatic ductal adenocarcinoma: hsa_circ_0001649. Gene. 2018;675:88-93. doi:10.1016/j.gene.2018.06.099

72. Zhang X, Tan P, Zhuang Y, Du L. Hsa_circRNA_001587 upregulates SLC4A4 expression to inhibit migration, invasion and angiogenesis of pancreatic cancer cells via binding to microRNA-223. Am J Physiol Gastrointest Liver Physiol. 2020;319(6):G703-G717. doi:10.1152/ajpgi.00118.2020

73. Wong $\mathrm{CH}$, Lou UK, Li Y, et al. CircFOXK2 promotes growth and metastasis of pancreatic ductal adenocarcinoma by complexing with RNA-binding proteins and sponging MiR-942. Cancer Res. 2020;80(11):2138-2149. doi:10.1158/0008-5472. CAN-19-3268

74. Lu J, Li X, Wang F, et al. YB-1 expression promotes pancreatic cancer metastasis that is inhibited by microRNA-216a. Exp Cell Res. 2017;359(2):319-326. doi:10.1016/j.yexcr.2017.07.039

75. Lim JP, Nair S, Shyamasundar S, et al. Silencing Y-box binding protein-1 inhibits triple-negative breast cancer cell invasiveness via regulation of MMP1 and beta-catenin expression. Cancer Lett. 2019;452:119-131. doi:10.1016/j.canlet.2019.03.014

76. Chua PJ, Lim JP, Guo TT, et al. Y-box binding protein-1 and STAT3 independently regulate ATP-binding cassette transporters in the chemoresistance of gastric cancer cells. Int J Oncol. 2018;53(6):2579-2589. doi:10.3892/ijo.2018.4557
77. Ye Z, Zhu Z, Xie J, et al. Hsa_circ_0000069 knockdown inhibits tumorigenesis and exosomes with downregulated hsa_circ_0000069 suppress malignant transformation via inhibition of STIL in pancreatic cancer. Int $J$ Nanomedicine. 2020;15:9859-9873. doi:10.2147/IJN.S279258

78. Ojha R, Nandani R, Chatterjee N, Prajapati VK. Emerging role of circular RNAs as potential biomarkers for the diagnosis of human diseases. Adv Exp Med Biol. 2018;1087:141-157.

79. Wang Y, Li Z, Xu S, Guo J. Novel potential tumor biomarkers: circular RNAs and exosomal circular RNAs in gastrointestinal malignancies. J Clin Lab Anal. 2020;34(7):e23359.

80. Xu Y, Yao Y, Gao P, Cui Y. Upregulated circular RNA circ_0030235 predicts unfavorable prognosis in pancreatic ductal adenocarcinoma and facilitates cell progression by sponging miR-1253 and miR-1294. Biochem Biophys Res Commun. 2019;509(1):138-142. doi:10.1016/j.bbrc.2018.12.088

81. Hao L, Rong W, Bai L, et al. Upregulated circular RNA circ_0007534 indicates an unfavorable prognosis in pancreatic ductal adenocarcinoma and regulates cell proliferation, apoptosis, and invasion by sponging miR-625 and miR-892b. J Cell Biochem. 2019;120(3):3780-3789. doi:10.1002/jcb.27658

82. Yang F, Liu DY, Guo JT, et al. Circular RNA circ-LDLRAD3 as a biomarker in diagnosis of pancreatic cancer. World J Gastroenterol. 2017;23(47):8345-8354. doi:10.3748/wjg.v23.i47.8345

83. Kristensen LS, Hansen TB, Venø MT, Kjems J. Circular RNAs in cancer: opportunities and challenges in the field. Oncogene. 2018;37(5):555-565. doi:10.1038/onc.2017.361

84. Verduci L, Ferraiuolo M, Sacconi A, et al. The oncogenic role of circPVT1 in head and neck squamous cell carcinoma is mediated through the mutant p53/YAP/TEAD transcription-competent complex. Genome Biol. 2017;18(1):237. doi:10.1186/s13059-0171368-y

85. Piwecka M, Glažar P, Hernandez-Miranda LR, et al. Loss of a mammalian circular RNA locus causes miRNA deregulation and affects brain function. Science. 2017;357:6357. doi:10.1126/ science.aam8526

86. Wang YZ, An Y, Li BQ, Lu J, Guo JC. Research progress on circularRNAs in pancreatic cancer: emerging but promising. Cancer Biol Ther. 2019;20(9):1163-1171. doi:10.1080/ 15384047.2019.1617563

87. Xiao Y. Construction of a circRNA-miRNA-mRNA network to explore the pathogenesis and treatment of pancreatic ductal adenocarcinoma. $J$ Cell Biochem. 2020;121(1):394-406. doi:10.1002/jcb.29194

88. Xu C, Yu Y, Ding F. Microarray analysis of circular RNA expression profiles associated with gemcitabine resistance in pancreatic cancer cells. Oncol Rep. 2018;40(1):395-404. doi:10.3892/or.2018.6450

89. Shao F, Huang M, Meng F, Huang Q. Circular RNA signature predicts gemcitabine resistance of pancreatic ductal adenocarcinoma. Front Pharmacol. 2018;9:584. doi:10.3389/fphar.2018.00584

90. Liu Y, Xia L, Dong L, et al. CircHIPK3 promotes gemcitabine (GEM) resistance in pancreatic cancer cells by sponging miR-330-5p and targets RASSF1. Cancer Manag Res. 2020;12:921-929. doi:10.2147/CMAR.S239326

91. Wenzhe G, Jiahao X, Cheng P, Hongwei Z, Xiao Y. Circular RNA HIPK3 is a prognostic and clinicopathological predictor in malignant tumor patients. J Cancer. 2020;11(14):4230-4239. doi:10.7150/ jca.40001

92. An Y, Cai H, Zhang Y, et al. circZMYM2 competed endogenously with miR-335-5p to regulate JMJD2C in pancreatic cancer. Cell Physiol Biochem. 2018;51(5):2224-2236. doi:10.1159/ 000495868

93. Li Z, Yanfang W, Li J, et al. Tumor-released exosomal circular RNA PDE8A promotes invasive growth via the miR-338/ MACC1/MET pathway in pancreatic cancer. Cancer Lett. 2018;432:237-250. doi:10.1016/j.canlet.2018.04.035 
94. Yang J, Cong $X$, Ren $M$, et al. Circular RNA hsa_circRNA_0007334 is predicted to promote MMP7 and COL1A1 expression by functioning as a miRNA sponge in pancreatic ductal adenocarcinoma. J Oncol. 2019;2019:7630894. doi:10.1155/2019/7630894

95. Chen Y, Li Z, Zhang M, et al. Circ-ASH2L promotes tumor progression by sponging miR-34a to regulate Notch 1 in pancreatic ductal adenocarcinoma. J Exp Clin Cancer Res. 2019;38 (1):466. doi:10.1186/s13046-019-1436-0

96. Qu S, Hao X, Song W, et al. Circular RNA circRHOT1 is upregulated and promotes cell proliferation and invasion in pancreatic cancer. Epigenomics. 2019;11(1):53-63. doi:10.2217/epi2018-0051

97. Ling S, He Y, Li X, et al. CircRHOT1 mediated cell proliferation, apoptosis and invasion of pancreatic cancer cells by sponging miR-125a-3p. J Cell Mol Med. 2020;24(17):9881-9889. doi: $10.1111 /$ jcmm. 15572

98. Xing $\mathrm{C}$, Ye $\mathrm{H}$, Wang $\mathrm{W}$, et al. Circular RNA ADAM9 facilitates the malignant behaviours of pancreatic cancer by sponging miR-217 and upregulating PRSS3 expression. Artif Cells Nanomed Biotechnol. 2019;47(1):3920-3928. doi:10.1080/ 21691401.2019.1671856

99. Xiong X, Feng J, Yang X, et al. Circular RNA CDR1as promotes tumor progression by regulating miR-432-5p/E2F3 axis in pancreatic cancer. Cancer Cell Int. 2021;21(1):112. doi:10.1186/ s12935-021-01812-3

100. Yao J, Zhang C, Chen Y, Gao S. Downregulation of circular RNA circ-LDLRAD3 suppresses pancreatic cancer progression through miR-137-3p/PTN axis. Life Sci. 2019;239:116871. doi:10.1016/j. Ifs.2019.116871
101. Shi H, Li H, Zhen T, Dong Y, Pei X, Zhang X. hsa circ 001653 implicates in the development of pancreatic ductal adenocarcinoma by regulating microRNA-377-mediated HOXC6 axis. Mol Ther Nucleic Acids. 2020;20:252-264. doi:10.1016/j.omtn.2019.12.028

102. Guo W, Zhao L, Wei G, Liu P, Zhang Y, Fu L. Blocking circ_0013912 suppressed cell growth, migration and invasion of pancreatic ductal adenocarcinoma cells in vitro and in vivo partially through sponging miR-7-5p. Cancer Manag Res. 2020;12:7291-7303. doi:10.2147/CMAR.S255808

103. Xu S, Lei SL, Liu KJ, Yi SG, Yang ZL, Yao HL. circSFMBT1 promotes pancreatic cancer growth and metastasis via targeting miR-330-5p/PAK1 axis. Cancer Gene Ther. 2020.

104. Huang L, Han J, Yu H, et al. CircRNA_000864 upregulates B-cell translocation gene 2 expression and represses migration and invasion in pancreatic cancer cells by binding to miR-361-3p. Front Oncol. 2020;10:547942. doi:10.3389/fonc.2020.547942

105. Zhang T, Li M, Lu H, Peng T. Up-regulation of circEIF6 contributes to pancreatic cancer development through targeting miR-557/SLC7A11/PI3K/AKT signaling. Cancer Manag Res. 2021;13:247-258. doi:10.2147/CMAR.S280307

106. Shen P, Yang T, Chen Q, et al. CircNEIL3 regulatory loop promotes pancreatic ductal adenocarcinoma progression via miRNA sponging and A-to-I RNA-editing. Mol Cancer. 2021;20(1):51. doi:10.1186/s12943-021-01333-7

107. Han X, Fang Y, Chen P, et al. Upregulated circRNA hsa_circ_0071036 promotes tumourigenesis of pancreatic cancer by sponging miR-489 and predicts unfavorable characteristics and prognosis. Cell Cycle. 2021;20(4):369-382. doi:10.1080/ 15384101.2021.1874684

\section{Publish your work in this journal}

Cancer Management and Research is an international, peer-reviewed open access journal focusing on cancer research and the optimal use of preventative and integrated treatment interventions to achieve improved outcomes, enhanced survival and quality of life for the cancer patient.
The manuscript management system is completely online and includes a very quick and fair peer-review system, which is all easy to use. Visit http://www.dovepress.com/testimonials.php to read real quotes from published authors. 